\title{
Output Performance Analysis on a Two-degrees- of-Freedom Bistable Piezoelectric Vibration Generator
}

\author{
http://dx.doi.org/10.3991/ijoe.v11i6.5022 \\ Qing He, Xinhua Mao and Dongliang Chu \\ North China Electric Power University, Beijing, P.R. China
}

\begin{abstract}
A two-degrees-of-freedom bistable piezoelectric vibration generator was designed for harvesting lowfrequency and weak-amplitude vibration energy in a real environment. And a dynamic model of a piezoelectric vibration generator system was established. The influence of the external excitation on the output characteristics of the twodegrees-of-freedom piezoelectric vibration generator system was simulated and analyzed. The results show that the twodegrees-of-freedom bistable piezoelectric vibration generator would do better in harvesting and converting lowfrequency and weak-amplitude vibration energy.
\end{abstract}

Index Terms - vibration energy harvesting; weak amplitude; degrees of freedom; dynamic response; vibration performance

\section{INTRODUCTION}

The vibration energy is converted into electrical energy by a micro piezoelectric vibration generator based on the piezoelectric effect principle, which provides a new power supply mode for sensor network nodes and other electronic devices. It also solves the problem of chemical battery pollution and short-time power supply. The bistable piezoelectric vibration generator can effectively expand the resonance bandwidth of vibration excitation, and the vibration energy collection efficiency is improved. It has become a hot spot of research in the field of vibration energy harvesting. But the single-degree-of-freedom piezoelectric vibration generator system is limited by its amplitude threshold. Only when the excitation vibration amplitude is greater than the amplitude threshold of the system can the generator system produce the output response in a two-well potential. And with the increase of the excitation frequency, the amplitude threshold of the system also increases. It becomes difficult to convert the low-amplitude vibration energy in the real environment into electrical energy for a single-degree-of-freedom piezoelectric vibration generator.[1-7]

The authors designed a two-degrees-of-freedom bistable piezoelectric vibration generator based on the structures of the single-degree-of-freedom bistable piezoelectric vibration generator by adding an amplitude amplifier. The improved structure retains the bistable characteristics of the single-degree-of-freedom bistable piezoelectric vibration generator and also magnifies the amplitude of the excitation source. It becomes easier to harvest the lowamplitude vibration energy and make up the defect of the single-degree-of-freedom bistable piezoelectric vibration generator.

\section{STRUCTURE AND DYNAMIC MODEL OF THE SYSTEM}

\section{A. Basic structure}

The system structure of

a two-degree-of-freedom bistable piezoelectric vibration generator is shown in Figure 1.Compared with the structure of the single-degree-of-freedom bistable piezoelectric vibration generator system, the structure of the twodegree-of-freedom bistable piezoelectric vibration generator keeps the original magnetic structure, so the system still has bistable characteristics. At the same time the spring can amplify the amplitude of an excitation vibration source. This is equivalent to a larger-amplitude vibration source directly acting on the single-degree-offreedom bistable piezoelectric vibration generator. It will generate more power.[8-9]

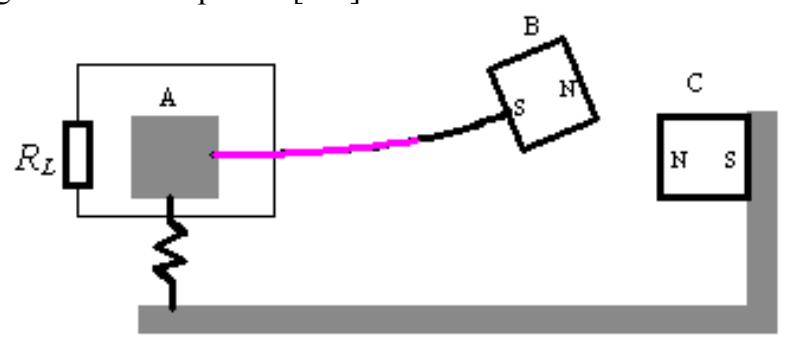

Figure 1. The structure of two-degree-freedom bistable piezoelectric vibration generator

\section{B. The lumped parameter model}

According to the structure of the two-single-degrees-offreedom bistable piezoelectric vibration generators, three assumptions follow for simplifying the analysis of the model. First, the link between the piezoelectric thin film and the metal elastic substrate is ideal bonding. Second, the link between the spring and the vibration table and between the spring and the base of the cantilever beam both are ideal bonding. Finally, neglecting the influence of gravity and the longitudinal movement of the spring, the vibration of the vertical plane is only considered.

The lumped parameter model of the two-singledegrees-of-freedom bistable piezoelectric vibration generator is shown in Figure 2. The piezoelectric generation systems are made of the cantilever beam, the piezoelectric ceramic, and the magnet at the end of the cantilever. The equivalent mass, equivalent damping, and equivalent stiffness of the system are, respectively, $M_{e q} 、 C_{e q}$. 
$K_{e q}$. The values can be determined by the following formula $^{[10]}$.

$$
\begin{gathered}
M_{e q}=\frac{33}{140}\left(m_{b}+m_{p}\right)+m_{c} \\
K_{e q}=\frac{E_{b} I_{b}}{L^{3}} \\
\alpha_{e q}=e_{31} \frac{h_{p} b_{p}}{L_{p}}
\end{gathered}
$$

Among $m_{b}, m_{p}, m_{c}$ are, respectively, the quality of the cantilever beam, the piezoelectric ceramic, and the magnet. $E_{b}, I_{b}, \mathrm{~L}$ are, respectively, the elastic modulus, the moment of inertia, and the length of the cantilever beam. $h_{p}, b_{p}, L_{p}$ are, respectively, the thickness, width, and length of the piezoelectric ceramic. $e_{31}$ is the piezoelectric constant.

A mass-spring-damping system is composed of a cantilever beam base " $A$ " and a spring. The equivalent mass, equivalent damping and equivalent stiffness of the system are, respectively, $M_{a}, C_{a}, K_{a}$. In the two-degree-offreedom system, the piezoelectric vibration generator is not only subjected to the elastic force and damping force of the cantilever beam, but also to the elastic force and damping force of the spring.

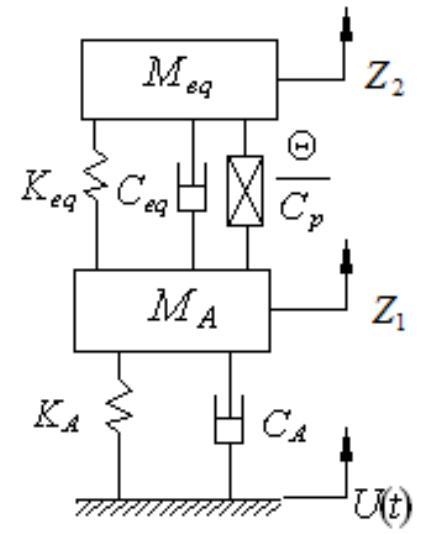

Figure 2. Electromechanical coupling model of the lumped parameters system

\section{Dynamic control equations}

According to the lumped parameter model, the external excitation force is acting on the spring and not the end of the cantilever beam. The equivalent mass, equivalent damping, equivalent stiffness, mechanical and electrical coupling force, and magnetic force of the cantilever beam piezoelectric vibration generator system in the twodegree-freedom system are the same as parameters of the single-degree-of-freedom in addition to the excitation part. According to the above analysis and Newton's law, the control equations of the two-degrees-of-freedom bistable piezoelectric vibration generator system are obtained.

$M_{e q} \ddot{Z}_{2}+C_{e q} \dot{Z}_{2}+K_{e q} Z_{2}-F_{g}\left(\frac{Z}{d}-\frac{Z^{3}}{2 d^{3}}\right)$
$-\alpha_{e q} \dot{V}=C_{e q} \dot{Z}_{1}+K_{e q} Z_{1}$

$$
\begin{aligned}
& C_{P} \dot{V}+\frac{V}{R_{L}}-\alpha_{e q} \dot{Z}_{2}=\mathbf{0} \\
& M_{a} \ddot{Z}_{1}+\left(C_{a}+C_{e q}\right) \dot{Z}_{1}-C_{e q} \dot{Z}_{2}+\left(K_{a}+K_{e q}\right) Z_{1} \\
& -K_{e q} Z_{2}=\left(M_{e q}+M_{a}\right) A \cos (\omega t)
\end{aligned}
$$

$F_{g}$ is the magnetic force. $A$ is the excitation amplitude . $\omega$ is the excitation frequency.

To facilitate the calculation and numerical simulation, the control equations of the system are processed by the dimensionless method.

$$
\begin{gathered}
\ddot{z}_{2}+\frac{C_{e q}}{M_{e q} \varpi_{2}} \dot{z}_{2}+\left(1-\frac{F_{g}}{d K_{e q}}\right) z_{2}+\frac{l^{2} F_{g}}{2 d^{3} K_{e q}} z_{2}^{3} \\
-\frac{\alpha e_{0}}{K_{e q} l} \dot{v}=\frac{C_{e q}}{M_{e q} \varpi_{2}} \dot{z}_{1}+\frac{K_{e q}}{M_{e q} \varpi_{2}^{2}} z_{1} \\
\dot{v}+\frac{\nu}{R_{L} C_{P}}-\frac{l \alpha}{e_{0} R C_{P}} \dot{z}_{2}=0 \\
\text { (8) } \\
-\frac{\ddot{z}_{1}+\frac{C_{a}+C_{e q}}{M_{a} \varpi_{1}} \dot{z}_{1}-\frac{C_{e q}}{M_{a} \varpi_{1}} \dot{z}_{2}+\frac{K_{a}+K_{e q}}{M_{a} \varpi_{1}^{2}} z_{1}}{M_{a} \varpi_{1}^{2}}=\frac{M_{a}+M_{e q}}{M_{a} l \varpi_{1}^{2}} A \cos \left(\omega \varpi_{1} t\right) \\
\text { Among, } Z_{2}=z_{2} l, t=\varpi_{1} \tau, Z_{1}=z_{1} l, \varpi_{1}=\sqrt{\frac{K_{a}}{M_{a}}} \\
\varpi_{2}=\sqrt{\frac{K_{e q}}{M_{e q}}, V=v e_{0} .}
\end{gathered}
$$$$
\text { (8) }
$$

Eq. (7), Eq. (8) and Eq. (9) are further simplified as follows.

$$
\begin{gathered}
\ddot{z}_{2}+2 \varsigma_{2} \dot{z}_{2}-\delta_{2} z_{2}+\gamma_{2} z_{2}^{3}-\lambda v=\varsigma_{2} \dot{z}_{1}+\kappa_{2} z_{1} \\
\dot{v}+\xi v-\sigma \dot{z}_{2}=0 \\
\ddot{z}_{1}+2 \varsigma_{1} \dot{z}_{1}-2 \eta_{1} \dot{z}_{2}+\delta_{1} z_{1}-\kappa_{1} z_{2}=f \cos (\Omega t) \\
\text { Among, } \varsigma_{2}=\frac{C_{e q}}{2 M_{e q} \varpi_{2}}, \delta_{2}=\frac{F_{g}}{d K_{e q}}-1, \gamma_{2}=\frac{l^{2} F_{g}}{2 d^{3} K_{e q}} \\
, \lambda=\frac{\alpha e_{0}}{K_{e q} l}, \kappa_{2}=\frac{K_{e q}}{M_{e q} \varpi_{2}^{2}}, \xi=\frac{v}{R_{L} C_{P}}, \sigma=\frac{l \alpha}{e_{0} R_{L} C_{P}}, \\
\varsigma_{1}=\frac{C_{a}+C_{e q}}{2 M_{a} \varpi_{1}}, \eta_{1}=\frac{C_{e q}}{2 M_{a} \varpi_{1}}, \delta_{1}=\frac{K_{a}+K_{e q}}{M_{a} \varpi_{1}^{2}} \\
\kappa_{1}=\frac{K_{e q}}{M_{a} \varpi_{1}^{2}}, f=\frac{M_{a}+M_{e q}}{M_{a} l \varpi_{1}^{2}} A, \Omega=\omega \varpi_{1} .
\end{gathered}
$$

The control equations of the system are transformed into state equations. 


$$
\left\{\begin{array}{l}
\dot{y}_{1}=y_{2} \\
\dot{y}_{2}=-2 \varsigma_{2} y_{2}+\delta_{2} y_{1}-\gamma y_{1}^{3}+\lambda y_{3}+\eta_{1} y_{5}+\kappa_{1} y_{4} \\
\dot{y}_{3}=-\xi y_{3}+\sigma y_{1} \\
\dot{y}_{4}=y_{5} \\
\dot{y}_{5}=-2 \varsigma_{1} y_{5}+2 \eta_{3} y_{2}-\delta_{1} y_{4}+\kappa_{2} y_{1}+f \cos (\Omega t)
\end{array}\right.
$$

Among, $y_{1}=z_{2}, y_{2}=\dot{z}_{2}, y_{3}=v, y_{4}=z_{1}, y_{5}=\dot{z}_{1}$.

\section{NUMERICAL ANALYSIS AND SIMULATION}

The structural parameters and the material physical properties of the two-degrees-of-freedom bistable piezoelectric vibration generator are shown in Table 1. According to Eq. (13), the output characteristics of the piezoelectric vibration generator system can be simulated.

TABLE I. PARAMETERS OF THE PIEZOELECTRIC VIBRATION GENERATOR

\begin{tabular}{lllll}
\hline parameters & $\begin{array}{l}\text { Cantilever } \\
\text { beam }\end{array}$ & PZT-5H & Magnet & Base \\
\hline $\begin{array}{l}\text { Density( } \\
\left.\mathrm{kg} / \mathrm{m}^{3}\right)\end{array}$ & 8860 & 7600 & 7500 & 7800 \\
Length(mm) & 75 & 45 & 15 & 35 \\
Width(mm) & 15 & 15 & 15 & 20 \\
$\begin{array}{l}\text { Thickness(mm) } \\
\text { Elasticity mod- } \\
\text { ulus }(\mathrm{GPa})\end{array}$ & 0.5 & 0.3 & 10 & 4 \\
$\begin{array}{l}\text { Piezoelectric } \\
\text { constant }\end{array}$ & 100 & 66 & & \\
$\begin{array}{l}\text { Dielectric } \\
\text { constant }\end{array}$ & & -5.35 & & \\
\hline
\end{tabular}

\section{A. Analysis of the influence of the excitation frequency on the response of the system.}

The frequency bifurcation diagrams are shown in Figure 3 when $f=0.123, f=0.223, f=0.323$, and $f=0.408$. According to Fig.3, with the increase of the excitation frequency, the two-degrees-of-freedom bistable piezoelectric vibration generator system has experienced a slight movement in a single potential well, another large jump movement between two potential wells, and finally back to a slight movement in a single potential well. The greater the excitation amplitude, the larger the resonant bandwidth of the system is. This shows that the system of the two-degrees-of-freedom bistable piezoelectric vibration generator has the same the upper-limit frequency threshold and the lower-limit frequency threshold. Compared with frequency bifurcation diagrams of the singledegree-of-freedom bistable piezoelectric vibration generator in the paper, the frequency resonant bandwidth of the two-degrees-of-freedom bistable piezoelectric vibration generator is greater than the single-degree-of-freedom system. And the two-degrees-of-freedom bistable piezoelectric vibration generator system more easily produces large jump movement with low frequency excitation.

\section{B. Analysis the influence of the excitation amplitude on the response of the system}

The amplitude bifurcation diagrams are shown in Figure 4 when $\Omega=0.3, \Omega=0.5, \Omega=0.9, \Omega=1.2$. According to Fig.4, when the excitation amplitude is greater than the amplitude threshold of the system, the twodegree-freedom bistable piezoelectric vibration generator would produce large jump movement between two potential wells, and with the increase of excitation amplitude, the output amplitude of the system has no obvious change. Analysis Fig.4(a) (b) shows that the higher the frequency, the smaller the amplitude of the threshold system is. Compared with amplitude bifurcation diagrams of the single-degree-of-freedom bistable piezoelectric vibration generator in the paper, the two-degrees-of-freedom bistable piezoelectric vibration generator system has a lowerlimit amplitude threshold. And with the same excitation frequency, the amplitude threshold of the two-degrees-offreedom bistable piezoelectric vibration generator system is smaller.

\section{Analysis the influence of the spring rigidity on the output characteristics}

The influence of the spring rigidity on the output amplitude of the piezoelectric vibration generator is shown in Figure 5 with different excitation amplitudes. According to Fig.5, with the other structural parameters and excitation conditions being the same, the greater the spring stiffness and the smaller the output amplitude of the piezoelectric vibration generator is. When the excitation amplitude is small, the output amplitude of the piezoelectric vibration generator is greatly influenced by the spring stiffness. When the excitation amplitude is large, the spring stiffness has little effect on the output amplitude of the piezoelectric vibration generator.

\section{EXPERIMENTAL RESULTS}

The experimental principle of the two-degrees-of-freedom bistable piezoelectric vibration generator is shown in Figure 6. Two acceleration sensors were posted on the vibration table and the magnet at the end of the cantilever beam, respectively. These were used to measure the excitation amplitude and the output amplitude of the piezoelectric vibration generator. Data from the sensor are transmitted to a computer through a data acquisition card. The amplitude and frequency of the excitation source are respectively controlled by a power amplifier and a signal generator.

The output effective amplitude of the bistable piezoelectric generator with different excitation frequency is shown in Figure 7. Both single-degree-of-freedom and two-degrees-of-freedom bistable piezoelectric generators have a frequency threshold. Compared with the singledegree-of-freedom bistable piezoelectric vibration generator, with an increase of excitation frequency, the twodegrees-of-freedom-bistable piezoelectric vibration generator preferred to produce a large output amplitude under the same amplitude excitation and with larger resonance bandwidth. This shows that the two-degrees-of-freedom bistable piezoelectric vibration generator is easier for collecting low-frequency vibration energy.

The output effective amplitude of the bistable piezoelectric generator with different excitation amplitude is shown in Figure 8. Both single-degree-of-freedom and 
two-degrees-of-freedom bistable piezoelectric generators have an amplitude threshold and only the lower-limit value. Compared with the single-degree-of-freedom bistable piezoelectric vibration generator, with an increase of excitation amplitude, the two-degrees-of-freedom-bistable piezoelectric vibration generator preferred to produce a large output amplitude under the same frequency excitation. This shows that the two-degrees-of-freedom bistable piezoelectric vibration generator is easier for collecting the low-amplitude vibration energy.

\section{CONCLUSION}

The two-degrees-of-freedom transducer structure was designed and the dynamic model of the generator system was established. The output characteristics of the generator were analyzed by simulation and experiment. The results are as follows.

First, with the same characteristics of a single-degreeof-freedom bistable generator vibration generator, the two-degrees-of-freedom bistable piezoelectric vibration generator has a frequency threshold and amplitude threshold. Second, under low frequency and low amplitude excitation, the two-degrees-of-freedom bistable piezoelectric vibration generator can produce a larger output amplitude. That would effectively improve the vibration energy and harvesting efficiency of the low frequency and low amplitude. And it also explains the shortage of the singledegree-of-freedom bistable piezoelectric vibration generator for not collecting low amplitude vibration energy.

\section{REFERENCES}

[1] Qing He, Xinhua Mao, and Dongliang Chu, "Study of micro piezoelectric vibration generator with added mass and capacitance suitable for broadband vibration”, AIP Advances, vol 5, 2015. http://dx.doi.org/10.1063/1.4926432

[2] Yuan Jiangbo, Xie Tao, Shan Xiaobiao, et al, "Vibrated model and experiments of multiple piezoelectric cantilevers in energy harvesting", Journal of Mechanical Engineering, vol. 46,no. 9,pp. 8792,2010. http://dx.doi.org/10.3901/JME.2010.09.087

[3] RL Harn and KW Wang, "A review of the recent research on vibration energy harvesting via bistable systems," Smart Mater. Struct, vol. 22, no.2, pp. 1330-1334, 2013. http://dx.doi.org/10.1088/0964-1726/22/2/023001

[4] MG Tehrani and SJ Elliott, "Extending the dynamic range of an energy harvester using nonlinear damping", Journal of Sound and Vibration, vol. 333, no.3, pp. 623-629, 2014. http://dx.doi.org/10.1016/j.jsv.2013.09.035
[5] YJ Gao, YG Leng, SB Fan, et al, "Studies on vibration response and energy harvesting of elastic-supported bistable piezoelectric cantilever beams", Acta Phys. Sin, vol. 63, no.9, pp. 090501-972, 2014.http://wulixb.iphy.ac.cn/EN/10.7498/aps.63.090501.

[6] Y Han, S Cao, S Sun, et al, "Response analysis of bistable piezoelectric cantilever beam considering geometric nonlinearity," Piezoelectrics \&Acoustooptics, vol. 36, no.1, pp. 132-139, 2014.

[7] Weiqun Liu, Fabien Formosa, Adrien Badel, et al, "Self-powered nonlinear harvesting circuit with a mechanical switch structure for a bistable generator with stoppers," Sensors and Actuators A: Physical, vol. 216, pp. 106-115, 2014. http://dx.doi.org/10.1016/ j.sna.2014.04.040

[8] H Qing, M Xinhua, and C Dongliang, "Dynamical characteristics of the bistable piezoelectric vibration generator under the random excitation," Noise and Vibration Control, vol. 35, no.2, pp. 36-40, 2015.

[9] W Yongguang, Z Yongzheng, J Wenping, et al, "Model and Experiments of a Broadband Piezoelectric Vibration Energy Harvester," Journal of Mechanical Engineering, vol. 51, no.6, pp. 155164, 2015. http://dx.doi.org/10.3901/JME.2015.06.155

[10] J Shangshuai, S Shu, and L Minggao, "Non-linear vibration analysis of bistable piezoelectric power generation system based on harmonic balance method," Journal of Vibration and Shock, vol. 33, no.6, pp. 170-173, 2014.

[11] X Mao, Q He, and T Huang, "Dynamic Modeling and Output Characteristic Analysis of a Micro Bistable Piezoelectric Generator", Key Engineering Materials, pp. 995-1003, 2015. http://dx.doi.org/10.4028/www.scientific.net/KEM.645-646.995

[12] Li Haitao and Qin Weiyang, "Bifurcation and chaos thresholds of bistable piezoelectric vibration energy harvesting systems", Applied Mathematics and Mechanics, vol. 35, no.6, pp. 652-666, 2014.

\section{AUTHORS}

Qing He is with the School of Energy, Power and Mechanical Engineering, North China Electric Power University, Beijing 102206, P.R. China. (email: hqng@163.com)

(Corresponding author) Xinhua Mao is with the School of Energy, Power and Mechanical Engineering, North China Electric Power University, Beijing 102206, P.R. China. (email: 30400414@qq.com)

Dongliang Chu is with the School of Energy, Power and Mechanical Engineering, North China Electric Power University, Beijing 102206, P.R. China. (email: 569256386@qq.com)

This work was supported in part by the Fundamental Research Funds for the Central Universities of China under grant no. 13XS13.

Submitted, 03 September, 2015. Published as resubmitted by the authors on 21 September, 2015. 


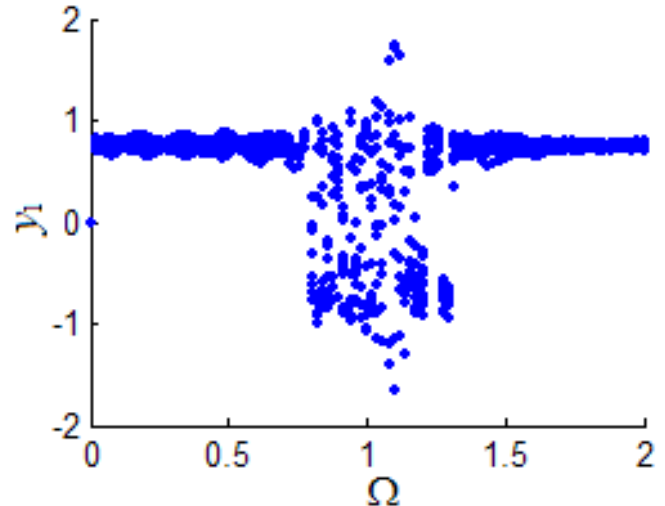

(a) $f=0.123$

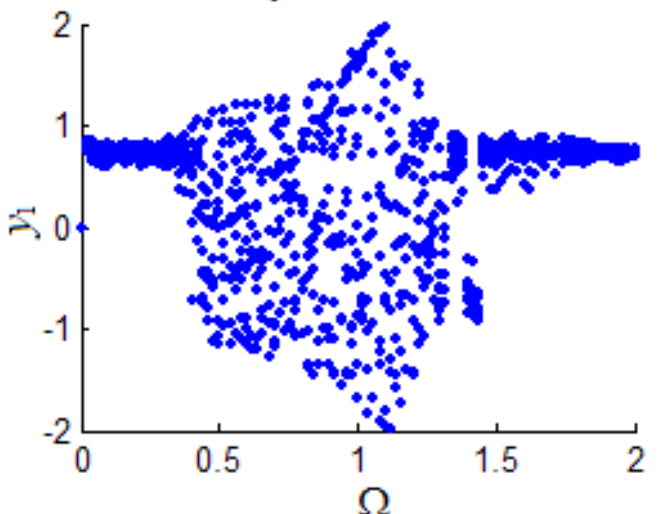

(c) $f=0.323$

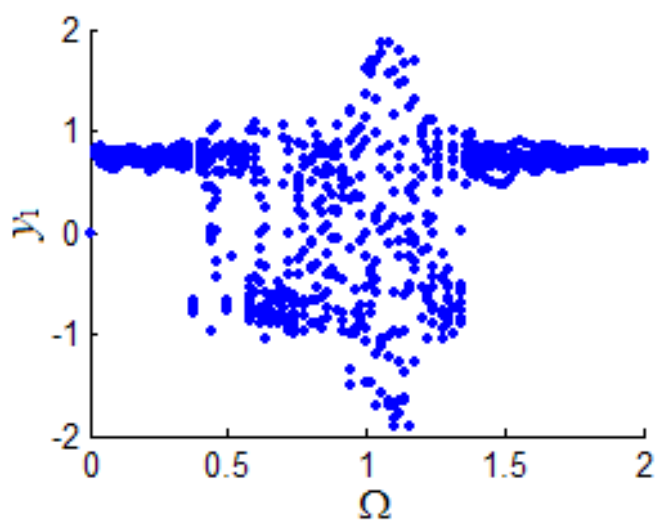

(b) $f=0.223$

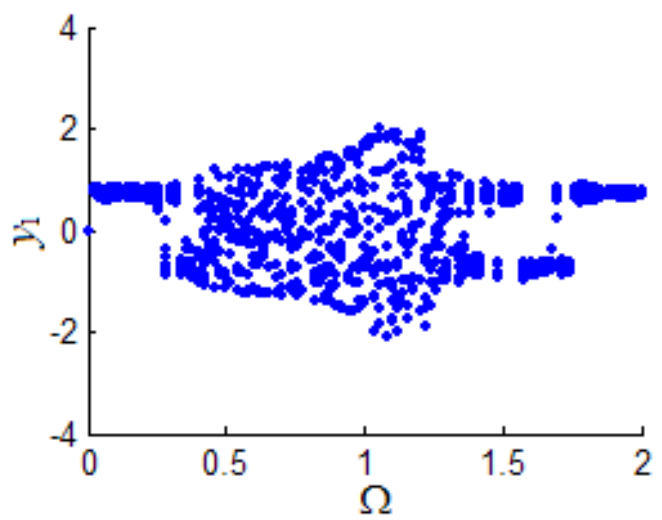

(d) $f=0.408$

Fig.3 Frequency bifurcation diagram with different amplitude

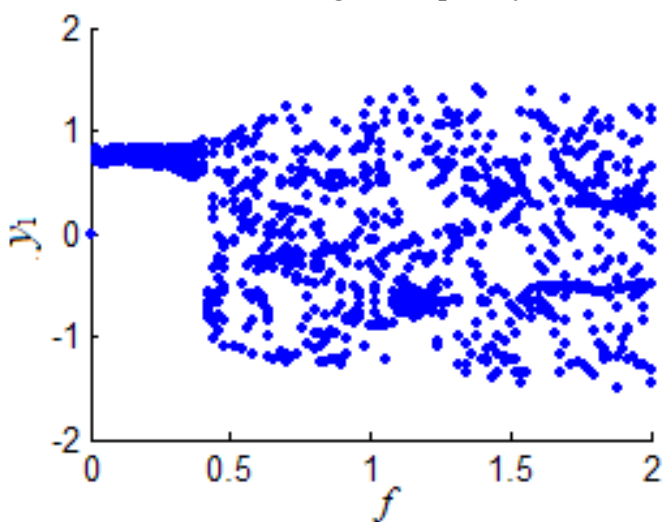

(a) $\Omega=0.3$

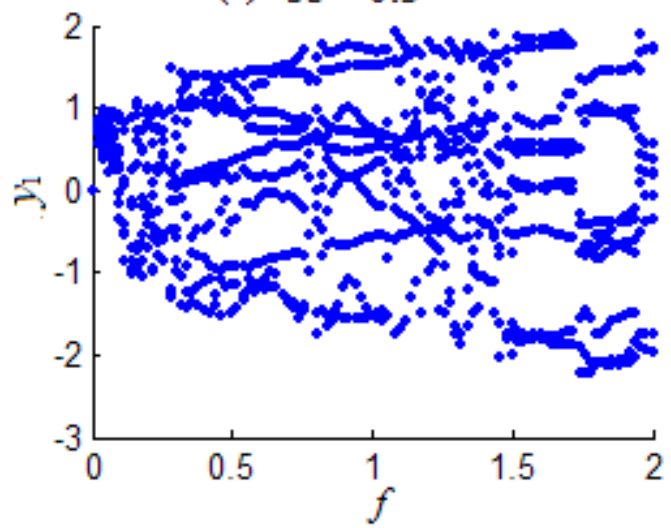

(c) $\Omega=0.9$

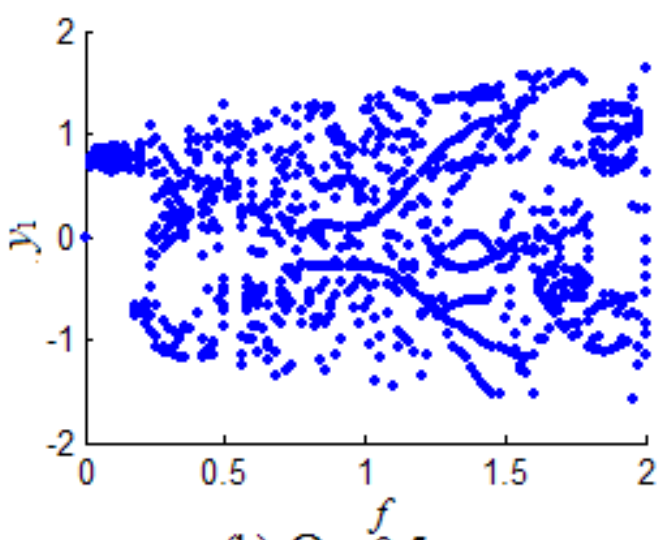

(b) $\Omega=0.5$

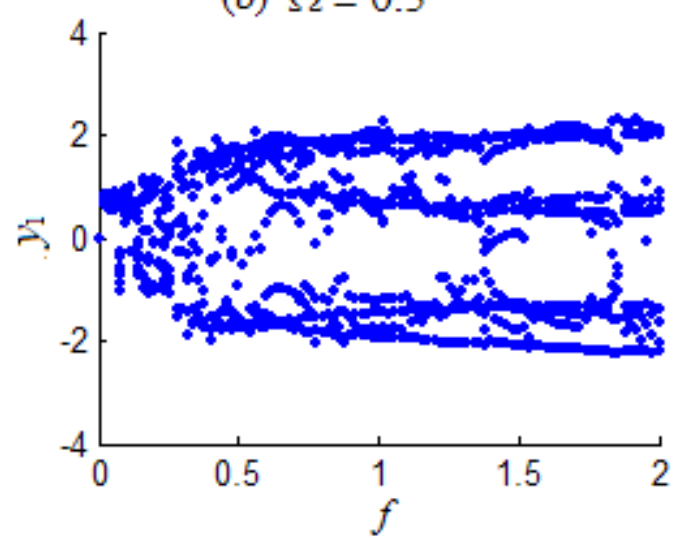

(d) $\Omega=1.2$

Fig.4 Amplitude bifurcation diagram with different frequency 


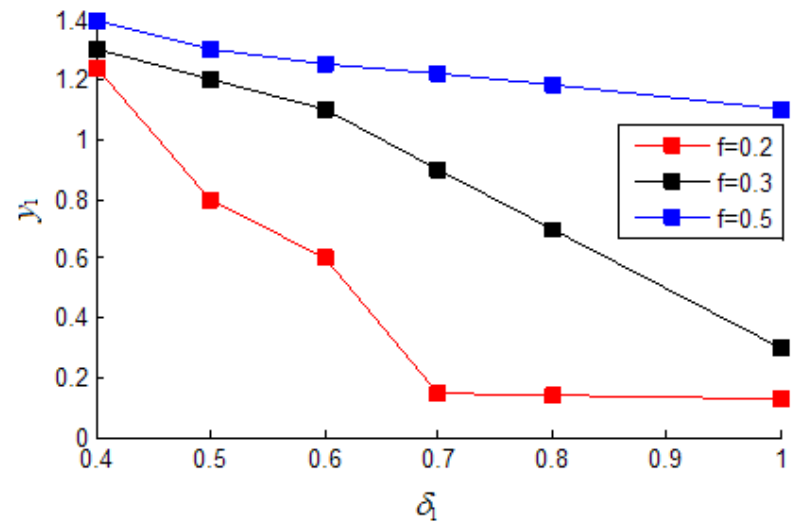

Fig.5 Relationship between spring rigidity coefficient and output amplitude

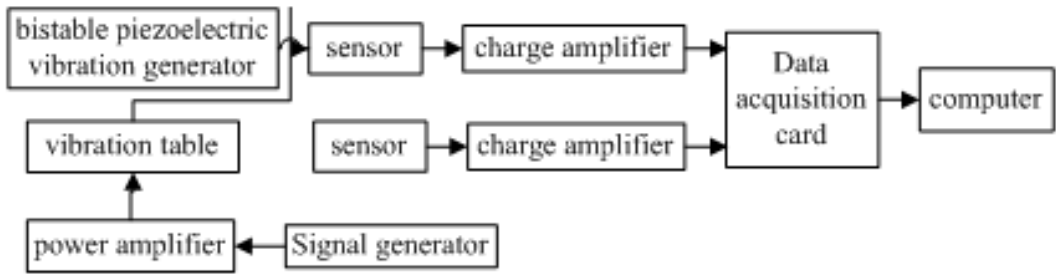

Fig.6 Experiment principle

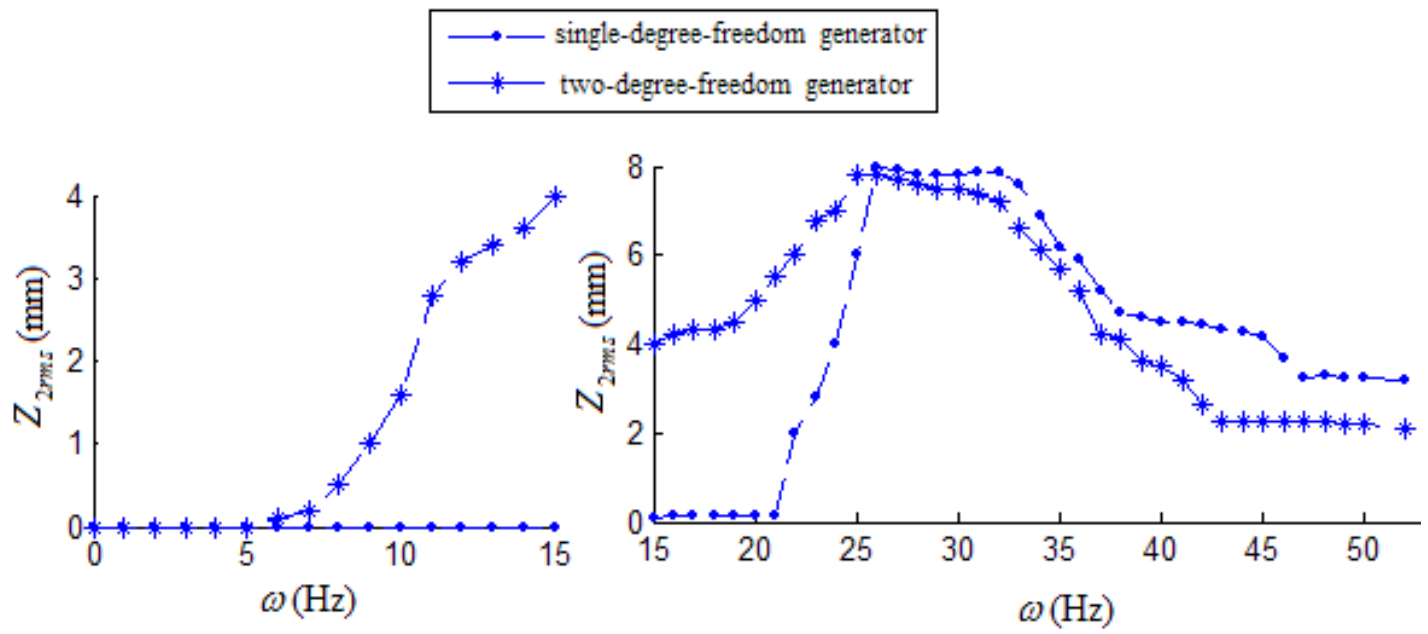

(a) $\omega=0 \sim 15$

(b) $\omega=15 \sim 52$

Fig.7 Output response curve when frequency changes

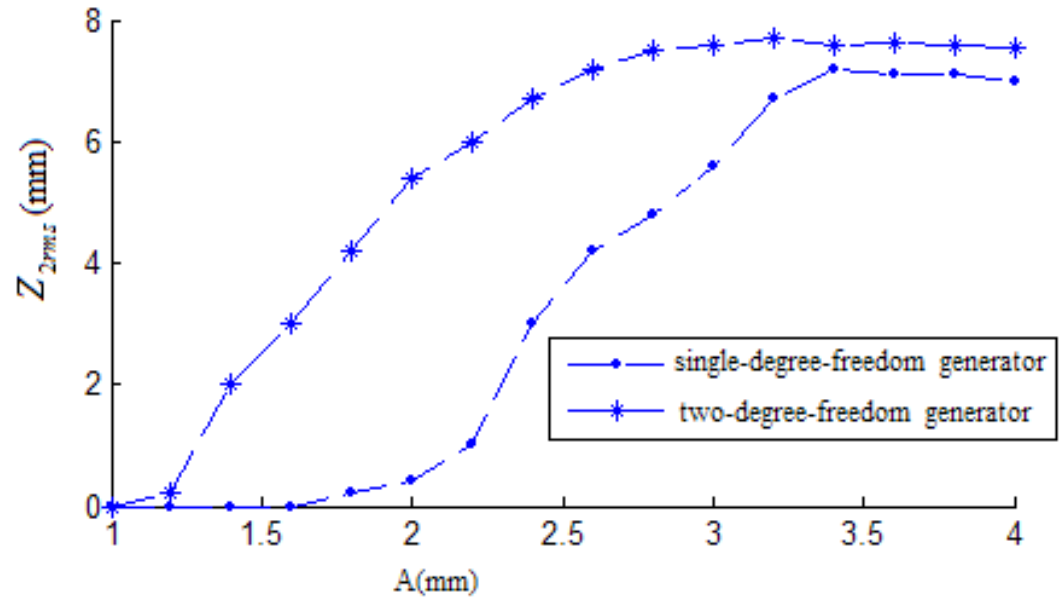

Fig.8 Output response curve when amplitude changes 